\title{
Does Immunomodulation Therapy of Sepsis Refer Directly to Tumor
}

\section{Immunotherapy?}

\section{Bailin Niu}

Department of critical care medicine, the first affiliated hospital of chongqing medical university, Chongqing, China

Corresponding Author: Bailin Niu, Department of critical care medicine, the first affiliated hospital of chongqing medical university, Chongqing, China E-mail: mailto:nberlin@126.com

Received date: August 03, 2019; Accepted date: August 14, 2019; Published date: August 20,2019

Citation: Niu B. (2019) Does Immunomodulation Therapy of Sepsis Refer Directly to Tumor Immunotherapy, J. Immunology and Inflammation Diseases Therapy. 2(3); Doi:10.31579/2637-8876/015

Copyright: ( $)$ 2019. Bailin Niu. This is an open-access article distributed under the terms of The Creative Commons Attribution License, which permits unrestricted use, distribution, and reproduction in any medium, provided the original author and source are credited

Sepsis remains a major cause of mortality in critical care, and the failures of highly touted trials have caused experts to call for re-evaluation of the approach toward sepsis [1]. Improved treatment protocols and updated organ support equipment have resulted in the majority of patients surviving the initial 72 hours of sepsis only to succumb later in the time course of the disease[2,3], and patients surviving after hospital discharge were significantly lower in the sepsis group compared with the control group in a long-term follow-up study[4]. There is increasing evidence of immunosuppression problems in patients with sepsis after the acute phase $[5,6]$, and it is found that there are many similar mechanisms with tumor immune disorder. Particularly, the influence of multiple negative costimulatory molecules on $\mathrm{T}$ cell inhibition in tumor microenvironment is also found in patients with sepsis during the duration or later period of infection[3,7,8], and researchers are eager to explore effective immunotherapy through them. However, our understanding of the impact of these negative co-stimulatory molecules on the expression of the changes is very limited. In particular, the spatio-temporal expression and regulation of these negative costimulatory molecules, as well as their interactions, have not been sufficiently studied. Recently, more and more studies have focused on the interaction between negative costimulator LAG3 and PD-1 in malignant tumors, and indeed some important findings have been made [9-11]. Duo blockade of PD-1 and LAG3 can obtain stronger anti-tumor or anti-viral effects. However, after Professor Chen lieping found that the activation of LAG3 can resist the therapeutic effect of PD-1 blockade [12] the relationship between the two molecules requires more urgent research in the infection disease field. In sepsis, a disease with acute pathophysiological changes, the spatiotemporal expression of which also is different from that of tumor. Whether the immunosuppression mechanism between them can be fully shared remains to be further studied. Recently, our group analyzed the expression characteristics of LAG3 and PD-1 in peripheral blood T cells of patients with sepsis at different time points and the functions of $\mathrm{T}$ cells with different phenotypes through strict inclusion criteria. The Auctores Publishing - Volume3-01 www.auctoresonline.org Page - 1 following important findings were made: 1) In sepsis, LAG3 and PD-1 had unique expression characteristics in $\mathrm{T}$ cells, and the $\mathrm{T}$ cells that coexpress LAG3 and PD-1 were significantly exhausted; 2) The proportion of $\mathrm{T}$ cells coexpressing LAG3 and PD-1 was negatively correlated with the total number of lymphocytes and positively correlated with the SOFA score; 3) In septic patients, the higher the proportion of LAG3 and PD-1-coexpressing T cells was, the longer the hospital stay and the higher the mortality rate. These findings suggest that sepsis immune disorder and tumor immunosuppression indeed have some similarities but also have their own characteristics. Although it was a small sample size study, it was clearly proposed that the immunoregulatory treatment of sepsis should fully consider the stages of disease course and the different phenotypes of LAG3 and PD-1 and other comprehensive factors. As well as fully elucidating the synergistic mechanism of PD-1 and LAG3, it is of great significance for both immunoregulatory therapy of infection and tumor in the future.

\section{Reference}

1. R. S. Hotchkiss, G. Monneret and D. Payen (2013). Immunosuppression in sepsis: a novel understanding of the disorder and a new therapeutic approach. Lancet Infect Dis, 13(3), 260-268.

2. J. Cohen, S. Opal and T. Calandra (2012). Sepsis studies need new direction. Lancet Infect Dis, 12(7), 503-505.

3. R. S. Hotchkiss, G. Monneret and D. Payen (2013). Sepsis-induced immunosuppression: from cellular dysfunctions to immunotherapy. Nat Rev Immunol, 13(12), 862-874.

4. H. Abu-Kaf, Y. Mizrakli, V. Novack and J. Dreiher (2018). LongTerm Survival of Young Patients Surviving ICU Admission With Severe Sepsis. Crit Care Med, 46(8), 1269-1275.

5. S. Ono, H. Tsujimoto, S. Hiraki and S. Aosasa (2018). Mechanisms of sepsis-induced immunosuppression and immunological modification therapies for sepsis. Ann Gastroenterol Surg, 2(5), 351-358. 
6. N. K. Patil, J. K. Bohannon and E. R. Sherwood (2016). Immunotherapy: A promising approach to reverse sepsis-induced immunosuppression. Pharmacol Res, 111, 688-702.

7. R. de Pablo, J. Monserrat, A. Prieto and M. Alvarez-Mon (2014).Role of circulating lymphocytes in patients with sepsis. Biomed Res Int, 671087

8. E. Watanabe, L. K. Thampy and R. S. Hotchkiss (2018). Immunoadjuvant therapy in sepsis: novel strategies for immunosuppressive sepsis coming down the pike. Acute Med Surg, 5(4), 309-315.

9. L. P. Andrews, A. E. Marciscano, C. G. Drake and D. A. Vignali (2017). LAG3 (CD223) as a cancer immunotherapy target. Immunol Rev, 276(1), 80-96.
10. L. T. Nguyen and P. S. Ohashi (2015). Clinical blockade of PD1 and LAG3--potential mechanisms of action. Nat Rev Immunol, 15(1), 45-56.

11. M. Wierz, S. Pierson, L. Guyonnet, E. Viry, A. Lequeux. et al. (2018) Dual PD1/LAG3 immune checkpoint blockade limits tumor development in a murine model of chronic lymphocytic leukemia. blood, 131(14), 1617-1621.

12. J. Wang, M. F. Sanmamed, I. Datar, T. T. Su, L. Ji. et al. (2019) Fibrinogen-like Protein 1 Is a Major Immune Inhibitory Ligand of LAG-3. Cell, 176(1-2), 334-347.e12 